Effect of pressure on superconductivity in the indium-doped topological crystalline insulator SnTe

This content has been downloaded from IOPscience. Please scroll down to see the full text.

2015 J. Phys.: Condens. Matter 27242201

(http://iopscience.iop.org/0953-8984/27/24/242201)

View the table of contents for this issue, or go to the journal homepage for more

Download details:

IP Address: 14.139.60.97

This content was downloaded on $13 / 10 / 2015$ at $06: 18$

Please note that terms and conditions apply. 


\title{
Effect of pressure on superconductivity in the indium-doped topological crystalline insulator SnTe
}

\author{
V K Maurya ${ }^{1}$, R Jha ${ }^{2}$, Shruti $^{1}$, V P S Awana ${ }^{2}$ and S Patnaik ${ }^{1}$ \\ ${ }^{1}$ School of Physical Sciences, Jawaharlal Nehru University, New Delhi 110067, India \\ ${ }^{2}$ National Physical Laboratory, New Delhi 110012, India \\ E-mail: spatnaik@mail.jnu.ac.in
}

Received 9 March 2015, revised 13 April 2015

Accepted for publication 27 April 2015

Published 22 May 2015

\begin{abstract}
We report on the impact of hydrostatic pressure on the superconductivity of optimally (indium)-doped SnTe which is established to be derived from a topological crystalline insulating phase. Single crystals of $\mathrm{Sn}_{1-x} \mathrm{In}_{x}$ Te were synthesized by a modified Bridgman method that exhibited maximum superconducting $T_{\mathrm{c}}$ of $4.4 \mathrm{~K}$ for $x=0.5$. Hydrostatic pressure up to $2.5 \mathrm{GPa}$ was applied on the crystals of $\mathrm{Sn}_{0.5} \mathrm{In}_{0.5} \mathrm{Te}$, and electrical resistivity as a function of temperature and pressure was measured. We observed a decrease in the onset superconducting transition temperature from $4.4 \mathrm{~K}$ to $2.8 \mathrm{~K}$ on increasing pressure from ambient to $2.5 \mathrm{GPa}$. The normal state resistivity also decreased abruptly by an order of magnitude at $0.5 \mathrm{GPa}$ but for higher pressures, it decreased marginally. From onset, offset and zero resistivity values, $\mathrm{d} T_{\mathrm{c}} / \mathrm{d} P$ of $\sim-0.6 \mathrm{~K} \mathrm{GPa}^{-1}$ was confirmed. The low temperature normal state resistivity followed $T^{2}$ dependence suggesting Fermi liquid behaviour both for ambient and high pressure data. This increase in metallic characteristics accompanied by normal state Fermi liquid behaviour is in accordance with a 'dome structure' for $T_{\mathrm{c}}$ variation with varying carrier concentration.
\end{abstract}

Keywords: topological crystalline superconductor, superconductor phase diagram, high pressure measurements

(Some figures may appear in colour only in the online journal)

\section{Introduction}

Superconductors derived from topological insulators (TIs) and topological crystalline insulators (TCIs) have attracted considerable attention recently [1-3]. From a theoretical perspective they could bring to fruition the search for the elusive Majorana fermions (MFs) and from the technological point of view they are expected to have a significant impact on topological quantum computation $[4,5]$. An example is the indium (In)-doped many-valley semiconductor tintelluride ( $\mathrm{SnTe}$ ) where a maximum superconducting transition temperature of $\sim 4.4 \mathrm{~K}$ is reported for $x \sim 0.5$ in the series $\operatorname{Sn}_{1-x} \operatorname{In}_{x} \mathrm{Te}[3,6,7]$. Several theoretical studies and detailed angle-resolved photo-electron spectroscopic (ARPES) measurements have established SnTe to be a TCI phase due to the underlying mirror symmetry of its crystalline lattice $[8,9]$. Towards developing an understanding of how these bulk superconductors are different from BCS or cuprate superconductors, in this communication we report the effect of hydrostatic pressure on the superconducting properties of optimally doped $\mathrm{Sn}_{0.5} \mathrm{In}_{05} \mathrm{Te}$.

The basic premise of measurements under high pressure is that they can effectively tune the electronic and phononic band structure. This has led to the discovery of superconductivity 
in myriad compounds at high pressure [10-12]. With regard to optimally doped superconductors, such studies could reflect if there is a 'dome structure' associated with phase transitions vis á vis carrier concentration that relates to quantum criticality and correlation effects [13]. Further, if pressure could revert to the insulating bulk phase, then in principle one can have superconductor-topological insulator interfaces leading to the emergence of MFs. Moreover, a dome structure of increasing $T_{\mathrm{c}}$ dependence with varying concentration of indium up to $50 \%$ was indicated earlier in SnTe [6], but the compounds were reportedly multiphase beyond $x=0.5$ and therefore high pressure studies are essential to elucidate the full phase diagram of $\mathrm{Sn}_{1-x} \operatorname{In}_{x} \mathrm{Te}$.

In particular, topological surface states have been investigated in $\mathrm{Bi}_{2} \mathrm{Se}_{3}$ and $\mathrm{Bi}_{2} \mathrm{Te}_{3}$ which have been driven to a superconducting state with application of external pressure [10-12]. In undoped topological insulators, superconductivity is achieved at relatively high pressure. For example, $\mathrm{Bi}_{2} \mathrm{Se}_{3}$ shows a turnover from semiconducting to metallic behaviour at $\sim 8 \mathrm{GPa}$ accompanied by a structural phase transition [14]. Superconductivity appears in $\mathrm{Bi}_{2} \mathrm{Se}_{3}$ at $\sim 13.5 \mathrm{GPa}$ at a transition temperature of $0.5 \mathrm{~K}$, which gradually increases to a maximum of $7 \mathrm{~K}$ on increasing pressure up to $30 \mathrm{GPa}$. At higher pressures, the $T_{\mathrm{c}}$ remains almost constant up to $50 \mathrm{Gpa}$ [10]. $\mathrm{Bi}_{2} \mathrm{Te}_{3}$ is another topological insulator, for which superconductivity is reported around $3 \mathrm{~K}$ under $3 \mathrm{GPa}$ pressure that increases to $8 \mathrm{~K}$ at $15 \mathrm{GPa}$ and for further higher pressures the $T_{\mathrm{c}}$ exhibits a decreasing trend [12]. Like $\mathrm{Bi}_{2} \mathrm{Se}_{3}$, $\mathrm{Bi}_{2} \mathrm{Te}_{3}$ also undergoes several structural transformations from a rhombohedral (R-3m) phase to monoclinic $(\mathrm{C} 2 / \mathrm{m})$ at $3 \mathrm{GPa}$, to monoclinic $(\mathrm{C} 2 / \mathrm{c})$ at $8 \mathrm{GPa}$ and finally to a bcc $\mathrm{Im}-3 \mathrm{~m}$ structure above $16 \mathrm{GPa}$ [12].

Superconductors derived from topological insulators through intercalation have also been subjected to various pressure studies [15]. $\quad \mathrm{Cu}_{x} \mathrm{Bi}_{2} \mathrm{Se}_{3}$ is a well-known topological insulator-based superconductor with a maximum $T_{\mathrm{c}}$ of around $3.8 \mathrm{~K}$ [15]. Point contact spectra on the surface of $\mathrm{Cu}$ intercalated $\mathrm{Bi}_{2} \mathrm{Se}_{3}$ exhibit signs of unconventional superconductivity [16]. On applying pressure on $\mathrm{Cu}_{x} \mathrm{Bi}_{2} \mathrm{Se}_{3}$ a gradual decrease in the superconducting transition temperature is reported and as pressure is increased further superconductivity disappears at $\sim 6.3 \mathrm{GPa}[17]$. With regard to TCI systems, a first-principles study on SnTe predicts a maximum superconducting $T_{\mathrm{c}}=7.16 \mathrm{~K}$ by pressure tuning the electron-phonon coupling parameters in the bcc phase (Pm-3m) [18]. Further, very recently Wang et al have shown unconventional superconductivity in 3D Dirac semi-metal $\mathrm{Cd}_{3} \mathrm{As}_{2}$ which belongs to a new class of topological superconductors [19]. In this communication, we focus on the impact of pressure on superconducting and normal state conduction of the recently discovered optimally doped TCI superconductor $\mathrm{Sn}_{0.5} \mathrm{In}_{0.5} \mathrm{Te}$. We found that the superconducting transition temperature $\left(T_{\mathrm{c}}\right)$ decreased monotonically with pressure $\left(\sim 0.6 \mathrm{~K} \mathrm{GPa}^{-1}\right)$ and the normal state resistivity also decreased by an order of magnitude at $0.5 \mathrm{GPa}$. Such behaviour shows a surprising resemblance with the curious case of over-doped cuprates.

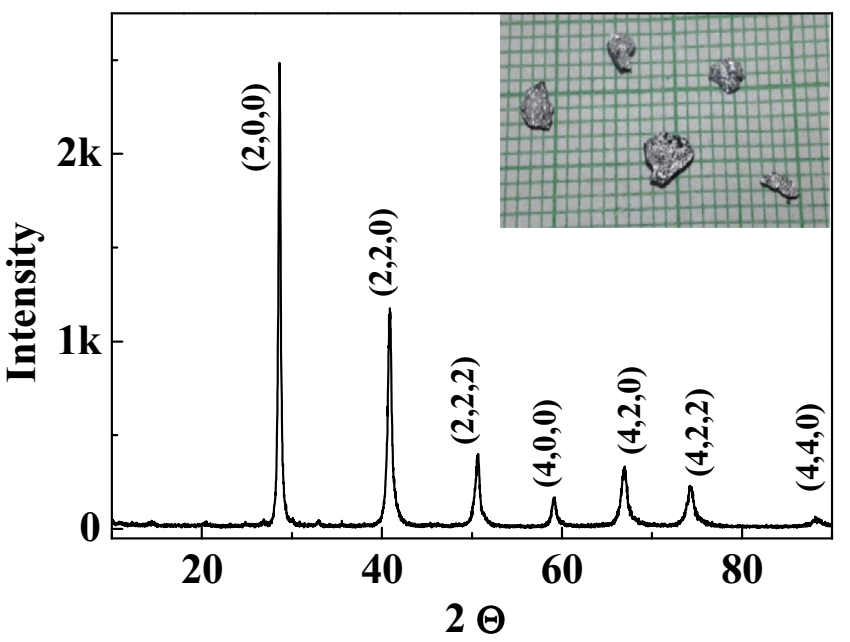

Figure 1. Powder XRD pattern of crystals of $S_{0.5} \mathrm{In}_{0.5}$ Te. The inset shows crystal flakes.

\section{Experimental methods}

Single crystals of $\mathrm{Sn}_{0.5} \operatorname{In}_{0.5} \mathrm{Te}$ were prepared by a modified Bridgman method. A series of compounds with varying indium concentration were prepared and optimum superconducting $T_{\mathrm{c}}$ was achieved for $\mathrm{Sn}_{0.5} \mathrm{In}_{0.5} \mathrm{Te}$ [7]. We studied the electrical resistivity at high pressure on this composition. Single crystals were obtained by melting stoichiometric amounts of high purity elemental powder of $\mathrm{Sn}(99.99 \%)$, Te (99.999\%) and shots of $\operatorname{In}(99.99 \%)$ at $900^{\circ} \mathrm{C}$ for $5 \mathrm{~d}$ in sealed evacuated quartz tubes. Intermittent shaking was performed for the homogeneity of the melt sample. The sample was cooled to $770^{\circ} \mathrm{C}$ over a period of $72 \mathrm{~h}$ followed by annealing at $770{ }^{\circ} \mathrm{C}$ for $48 \mathrm{~h}$. Silvery-shiny single crystals were cleaved along the $z$ axis. X-ray diffraction was carried out on the powdered samples by a $R I G A K U$ powder x-ray Diffractometer (Miniflex 600) [7]

Pressure-dependent resistivity measurements were performed in the Physical Property Measurements System (PPMS-14T, Quantum Design) using an HPC-33 Piston type pressure cell with Quantum Design DC resistivity option. Hydrostatic pressures were generated by a $\mathrm{BeCu} / \mathrm{NiCrAl}$ clamped piston-cylinder cell. The sample was immersed in a fluid (Daphne Oil) with pressure transmitting medium of Fluorinert in a Teflon cell. Annealed Pt wires were affixed to goldsputtered contact surfaces on each sample with silver epoxy in a four-probe configuration.

\section{Results and discussion}

The powder XRD pattern of $\mathrm{Sn}_{0.5} \mathrm{In}_{0.5}$ Te is shown in figure 1 . It confirms pure phase synthesis in agreement with reference data from JCPDF (No. 089-3974). The specimen crystallizes in a rock-salt structure with space group Fm-3m. The calculated lattice parameter is $\mathbf{a}=6.265 \AA$ and the cell volume is $245.65 \AA^{3}$. We note that previous data have reported continuance of the rock salt structure of SnTe up to $(x=0.5)[6,7]$. Further band structure calculations indicate that structural changes are expected only above $5 \mathrm{GPa}$ which is 


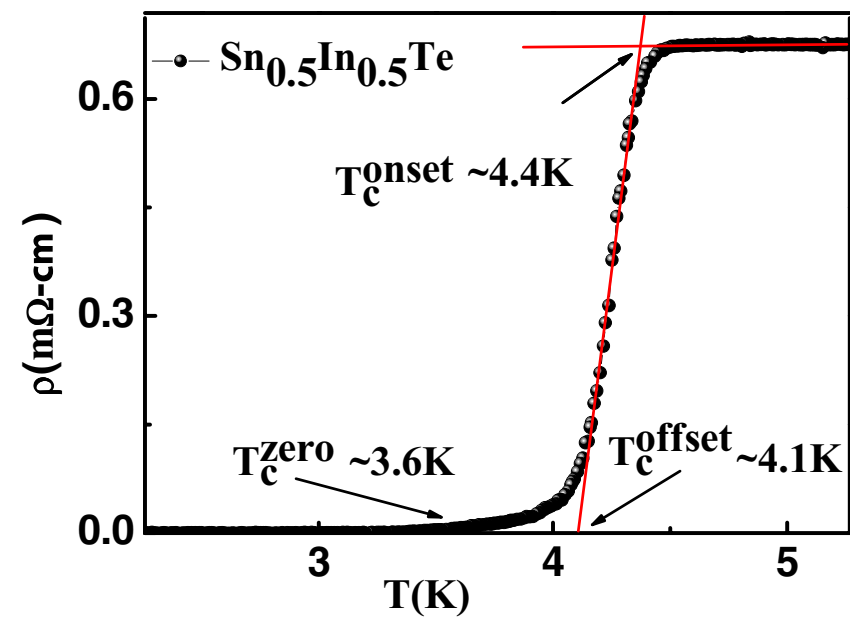

Figure 2. Resistive superconducting transition of $\operatorname{Sn}_{0.5} \mathrm{In}_{0.5} \mathrm{Te}$ at ambient pressure. $T_{\mathrm{c}}^{\text {onset }}, T_{\mathrm{c}}^{\text {offset }}$ and $T_{\mathrm{c}}^{\text {zero }}$ are indicated by arrows.

considerably above our experimental range [18]. From these accounts we infer that $x=0.5$ does not go into a new phase due either to pressure (less that $2.5 \mathrm{GPa}$ ) or dopant concentration. In the inset, as-grown crystal flakes are shown. The electrical resistivity as a function of temperature $(\rho-T)$ for $\mathrm{Sn}_{0.5} \mathrm{In}_{0.5} \mathrm{Te}$ at ambient pressure is shown in figure 2. The inset shows resistivity up to room temperature. We mark $T_{\mathrm{c}}^{\text {onset }}$ by the intersection of the two extrapolated lines, one corresponding to the superconducting transition line and the other being an extended normal state resistivity line. Similarly, $T_{\mathrm{c}}^{\text {offset }}$ is indicated by the intersection of the transition line and a zero resistivity line. We define $T_{\mathrm{c}}^{\text {zero }}$ as the temperature where a zero resistivity state was achieved. This is schematically shown in figure 2. From figure 2, The values of $T_{\mathrm{c}}^{\text {onset }}, T_{\mathrm{c}}^{\text {offset }}$ and $T_{\mathrm{c}}^{\text {zero }}$ for single-crystal $\mathrm{Sn}_{0.5} \mathrm{In}_{0.5} \mathrm{Te}$ are found to be $4.4 \mathrm{~K}, 4.1 \mathrm{~K}$ and $3.6 \mathrm{~K}$ respectively. The superconducting transition is sharp with a transition width of $\sim 0.3 \mathrm{~K}$.

The resistivity versus temperature behaviour near the superconducting transition $\left(T_{\mathrm{c}}\right)$ for varying pressure is shown in figure 3(a). For clarity, the data for ambient pressure are not included as they are an order of magnitude higher. It is seen that both the superconducting transition temperature and normal state resistivity decrease with increasing pressure. It can be seen that at a maximum pressure of $2.5 \mathrm{GPa}$, the $T_{\mathrm{c}}^{\text {onset }}$ decreases to $2.8 \mathrm{~K}$ from $4.4 \mathrm{~K}$ (ambient pressure), while the $T_{\mathrm{c}}^{\text {offset }}$ and $T_{\mathrm{c}}^{\text {zero }}$ decrease to $2.6 \mathrm{~K}$ and $2.3 \mathrm{~K}$ from $4.1 \mathrm{~K}$ and $3.6 \mathrm{~K}$ respectively. The $T_{\mathrm{c}}^{\text {onset }}, T_{\mathrm{c}}^{\text {offset }}$ and $T_{\mathrm{c}}^{\text {zero }}$ for $\mathrm{Sn}_{0.5} \operatorname{In}_{0.5} \mathrm{Te}$ superconductor at intermediate pressure are summarized in figure 3(b). It appears that the decrease in the $T_{\mathrm{c}}$ is approximately linear for two markers (onset, offset) of the superconducting transition. The negative coefficients of $T_{\mathrm{c}}$ suppression with pressure for three markers $\left(T_{\mathrm{c}}^{\text {onset }}, T_{\mathrm{c}}^{\text {offset }}\right.$ and $T_{\mathrm{c}}^{\text {zero }}$ ) are estimated to be $-0.66 \mathrm{~K} \mathrm{GPa}^{-1},-0.61 \mathrm{~K} \mathrm{GPa}^{-1}$ and $-0.57 \mathrm{~K} \mathrm{GPa}^{-1}$ respectively, and yield an average $\mathrm{d} T_{\mathrm{c}} / \mathrm{d} P$ of $-0.6 \mathrm{~K} \mathrm{GPa}^{-1}$ for the $\mathrm{Sn}_{0.5} \mathrm{In}_{0.5}$ Te superconductor. In fact, in conventional superconductors, it is very common to see the decreasing $T_{\mathrm{c}}$ trend as a function of increasing pressure. Sn, In and $\mathrm{Pb}$ all have negative $\mathrm{d} T_{\mathrm{c}} / \mathrm{d} P$ of $\sim 0.4 \mathrm{~K} \mathrm{GPa}^{-1}$ [20]. The principal reason for this decrease in simple metals is stiffening of the lattice and the consequent decrease in electron-phonon
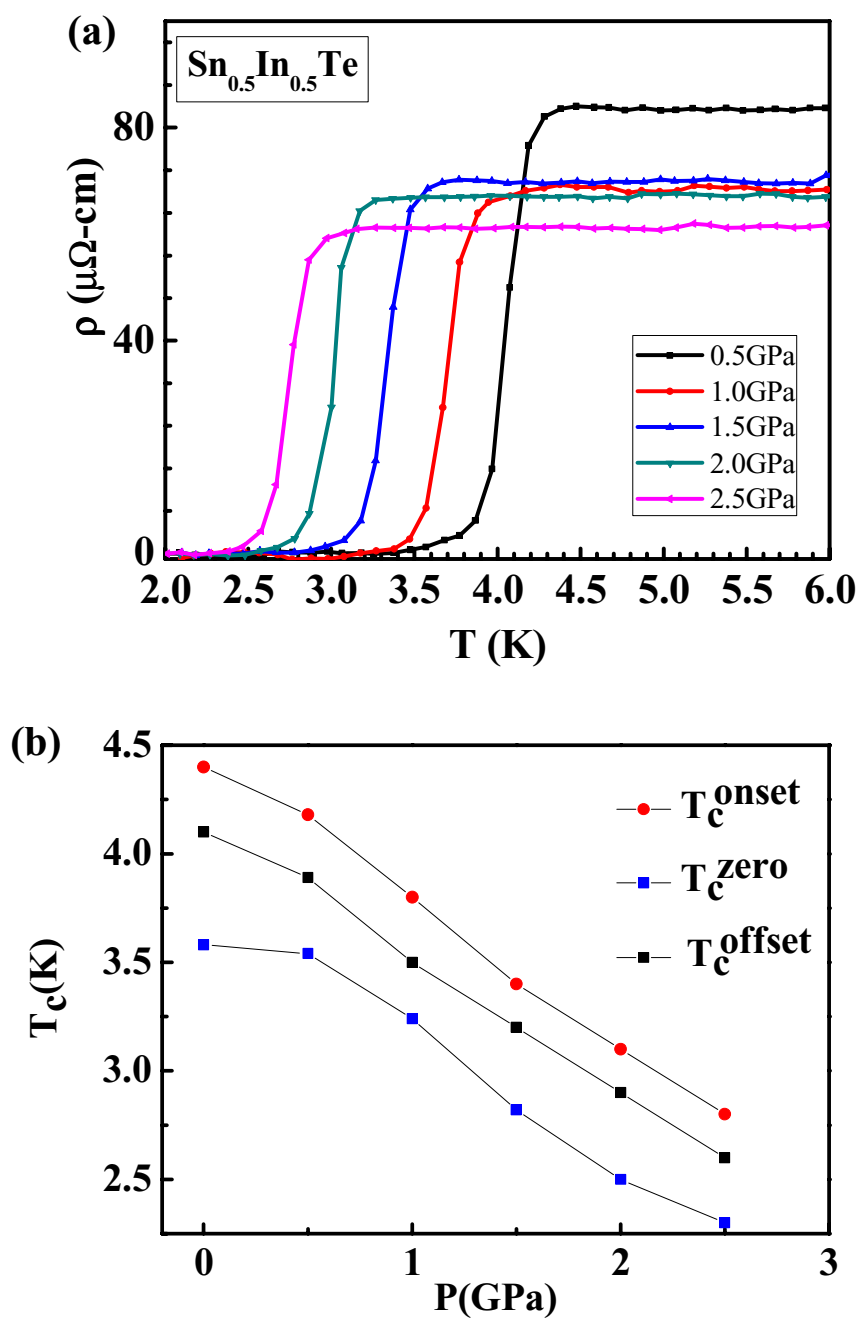

Figure 3. (a) Resistive superconducting transition of $\mathrm{Sn}_{0.5} \mathrm{In}_{0.5} \mathrm{Te}$ at different pressures from $0.5 \mathrm{GPa}$ to $2.5 \mathrm{GPa}$. An unambiguous decrease in superconducting transition temperature is seen. (b) Variation in superconducting $T_{\mathrm{c}}$ at various pressures for $\mathrm{Sn}_{0.5} \mathrm{In}_{0.5} \mathrm{Te}$. We can see a negative pressure coefficient $\left(\mathrm{d} T_{\mathrm{c}} / \mathrm{d} p\right)$ for all transitions $T_{\mathrm{c}}^{\text {onset }}, T_{\mathrm{c}}^{\text {offset }}$ and $T_{\mathrm{c}}^{\text {zero }}$.

coupling rather than electronic effects. On the other hand, for high $T_{\mathrm{c}}$ cuprates generally $T_{\mathrm{c}}(P)$ first increases with pressure and beyond a critical pressure it starts to decrease, exhibiting a dome structure in accordance with the $T_{\mathrm{c}}$ dependence on carrier concentration [21].

In figure 4(a) we compare resistivity versus temperature $(\rho-T)$ for $\mathrm{Sn}_{0.5} \mathrm{In}_{0.5} \mathrm{Te}$ taken at ambient pressure and applied pressures in the extended temperature range up to $250 \mathrm{~K}$. This is done to visualize the impact of hydrostatic pressure on the normal state conduction of $\operatorname{Sn}_{0.5} \operatorname{In}_{0.5} \mathrm{Te}$. We see that at applied pressure $0.5 \mathrm{GPa}$ the normal resistivity (resistivity just above the transition) decreases abruptly by nearly an order of magnitude. Quantitatively, a decrease of about 7.8 times $(659.76 \mu \Omega \mathrm{cm}-84.09 \mu \Omega \mathrm{cm})$ is observed. With further increase in pressure, while the normal state resistivity continues to decrease, the rate of change with pressure decreases substantially. Overall, the change in resistivity with temperature shows a metallic behaviour at both ambient and applied pressures up to $2.5 \mathrm{GPa}$ and clearly the 


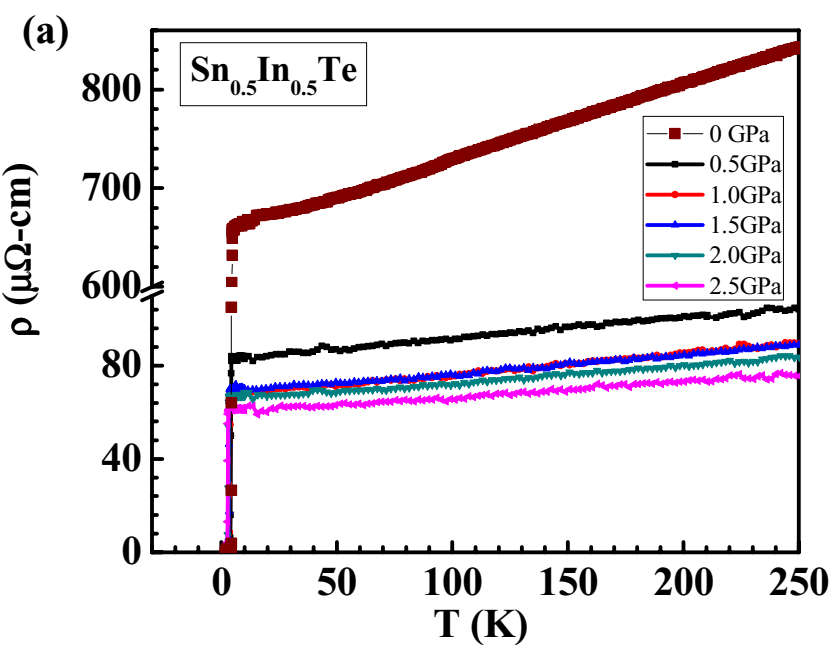

(b)

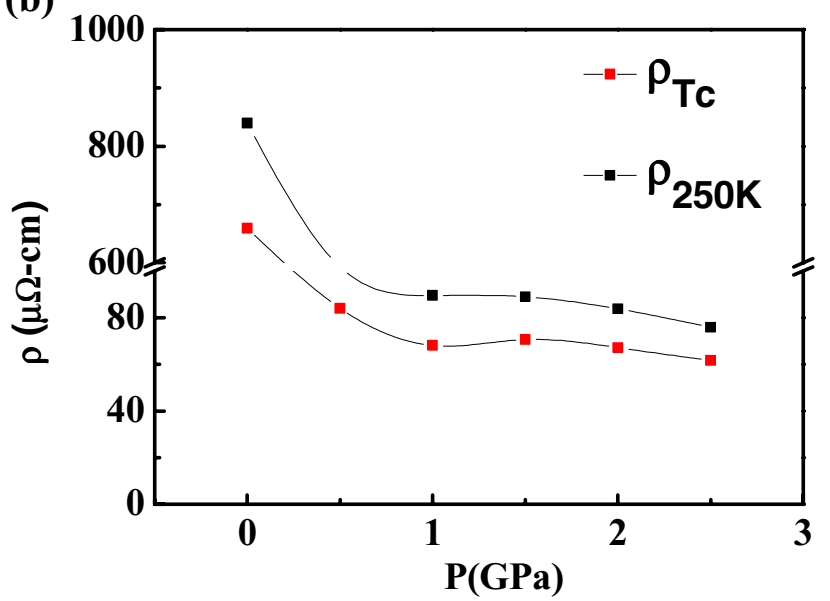

Figure 4. (a) Resistivity up to $250 \mathrm{~K}$ for $\mathrm{Sn}_{0.5} \mathrm{In}_{0.5} \mathrm{Te}$. A large decrease in resistivity is seen on application of $0.5 \mathrm{GPa}$. For higher pressure, resistivity decreases at a smaller rate. No significant variation in residual resistivity ratio is observed. (b) Magnitude of electrical resistivity at $250 \mathrm{~K}$ and at temperatures close to $T_{\mathrm{c}}$ plotted as a function of pressure. A sharp decrease in magnitude is observed at $0.5 \mathrm{GPa}$ followed by an almost linear decrease in the resistivity.

metallic behaviour increases with higher pressure. This is in contrast to $\mathrm{Cu}_{x} \mathrm{Bi}_{2} \mathrm{Se}_{3}$ where $\rho\left(T_{\mathrm{c}}\right)$ increases by 7 times at $2.31 \mathrm{GPa}$. For low carrier density superconductors the $\mathrm{BCS}$ equation relates superconducting transition temperature with carrier concentration; $T_{\mathrm{c}} \sim \theta_{\mathrm{D}} \exp \left(-1 / N\left(E_{\mathrm{F}}\right) V_{0}\right)$ where $\theta_{\mathrm{D}}$ is the Debye temperature, $V_{0}$ is the electronphonon coupling calliper and density of state $N\left(E_{\mathrm{F}}\right) \sim$ $m^{*} n^{1 / 3}$ which is a product of effective mass $\mathrm{m}^{*}$ and carrier concentration $n$. A decreasing carrier concentration (increasing normal state resistivity) with pressure can explain decreasing superconducting $T_{\mathrm{c}}$. But we find that normal state resistivity for $\mathrm{Sn}_{0.5} \mathrm{In}_{0.5} \mathrm{Te}$ decreases many-fold with pressure. Thus the controlling parameter for the $T_{\mathrm{c}}$ suppression mechanism seems to be decreasing effective mass $m^{*}$ in $\mathrm{Sn}_{0.5} \mathrm{In}_{0.5} \mathrm{Te}$ under a simplistic s-wave correlation [7]. In figure 4(b) we show the change in the resistivity just above the transition temperature and at $250 \mathrm{~K}$ for ambient and various applied pressures. We can see that both decrease very rapidly on application of $0.5 \mathrm{GPa}$ but for higher pressure, the change

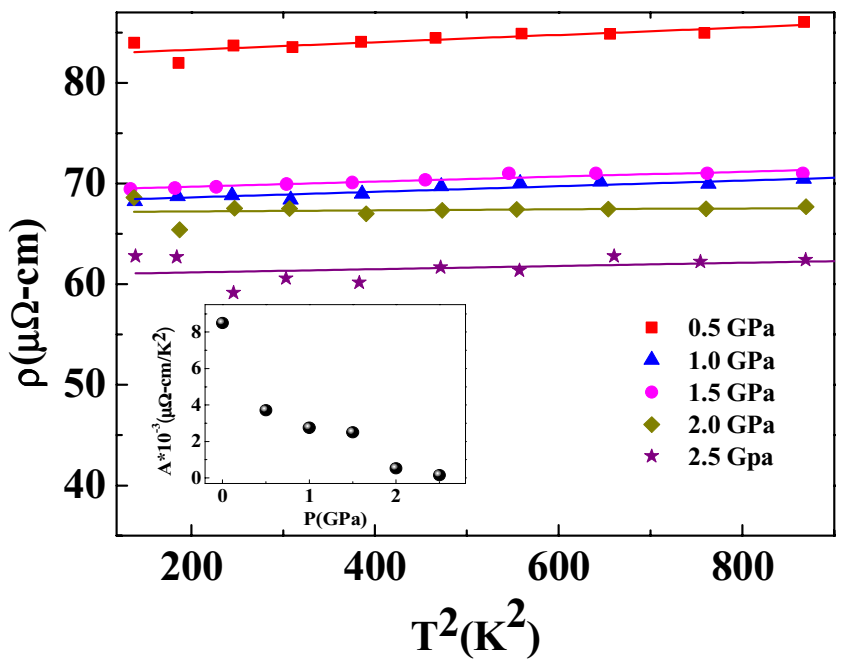

Figure 5. Resistivity versus $T^{2}$ for pressure $0,0.5,1,1.5,2$, $2.5 \mathrm{GPa}$. The solid lines are linear fits to the equation $\rho=\rho_{0}+A T^{2}$.

is relatively much smaller. The RRR (residual resistivity ratio) between resistivity at $250 \mathrm{~K}$ temperature and temperature just above the transition remains $\sim 1.25$ from ambient pressure to $2.5 \mathrm{GPa}$. This indicates that within experimental error, the impurity band contribution to the normal state conduction mechanism in $\mathrm{Sn}_{0.5} \mathrm{In}_{0.5} \mathrm{Te}$ remains mainly unaffected by applied pressure.

We note that both in high $T_{\mathrm{c}}$ cuprates and in pnictide superconductors, the transition temperature varies in a socalled dome structure as a function of doping concentration. In the overdoped region, the normal state resistivity is well characterized by Fermi liquid behaviour. To study the appropriateness of Fermi liquid theory (negligible electronic correlations), in figure 5 we plot the resistivity versus $T^{2}$ curves in the temperature range $11-30 \mathrm{~K}$ for $\mathrm{Sn}_{0.5} \mathrm{In}_{0.5} \mathrm{Te}$. In this theory, particularly with regard to heavy fermion systems, the strong interaction between charge carriers is replaced by weakly correlated quasi-particles with high effective mass. In figure 5, the resistivity data taken at various pressure are fitted to the equation $\rho=\rho_{0}+A T^{2}$ where $\rho_{0}$ relates to impurity scattering and the coefficient $A$ relates to square of the effective mass of the quasi-particles. The calculated values for $\rho_{0}$ and $\mathrm{A}$ are listed in table 1 . We find that for the pressure $0,0.5,1,1.5,2,2.5 \mathrm{GPa}$ the data follow a $T^{2}$ behaviour in the temperature range $11-30 \mathrm{~K}$ and the curves deviate from linearity above $30 \mathrm{~K}$. In the inset of figure 5 we plot the pressure dependence of coefficient A which indicates a decreasing trend with increasing pressure. This is suggestive of a weakening correlation between charge carriers in the overdoped region. But the origin of this correlation phenomenon in the semiconducting parent SnTe needs to be ascertained.

\section{Conclusion}

We have prepared single crystals of $\mathrm{Sn}_{0.5} \mathrm{In}_{0.5} \mathrm{Te}$ and applied pressures up to $2.5 \mathrm{GPa}$ to check their superconducting properties under pressure. This is an optimally doped 
Table 1. The values for $\rho_{0}$ and $A$ at various pressures for the equation $\rho=\rho_{0}+A T^{2}$.

\begin{tabular}{lllllll}
\hline Pressure $(\mathrm{GPa})$ & 0 & 0.5 & 1 & 1.5 & 2 & 2.5 \\
\hline$\rho_{0}(\mu \Omega \mathrm{cm})$ & 669.96 & 82.5 & 68.07 & 69.1 & 67.2 & 60.8 \\
$A\left(\mu \Omega \mathrm{cm} \mathrm{K}^{-2}\right)$ & $8.49 \times 10^{-3}$ & $3.71 \times 10^{-3}$ & $2.75 \times 10^{-3}$ & $2.49 \times 10^{-3}$ & $5.25 \times 10^{-4}$ & $1.58 \times 10^{-4}$ \\
\hline
\end{tabular}

specimen derived from a topological crystalline insulating phase. We found that superconducting $T_{\mathrm{c}}$ (onset) decreased with pressure from $4.4 \mathrm{~K}$ (ambient) to $3.8 \mathrm{~K}(2.5 \mathrm{GPa})$. This suppression of superconducting transition temperatures was found to be almost monotonic with pressure and the overall $\mathrm{d} T_{\mathrm{c}} / \mathrm{d} P$ was estimated to be $-0.6 \mathrm{~K} \mathrm{GPa}^{-1}$. Fermi liquid behaviour was indicated in the temperature range $11-30 \mathrm{~K}$ and we found that the normal state resistivity of the sample varied as a function of $T^{2}$ with increasing pressure. Such a systematic decrease with $T_{\mathrm{c}}$ with increasing metallicity and normal state $T^{2}$ behaviour is reminiscent of overdoped high $T_{\mathrm{c}}$ cuprates.

\section{Acknowledgments}

SP thanks DST-FIST and VPSA thanks DAE-SRC for supporting research infrastructure at JNU and NPL respectively. VM, Shruti and RJ acknowledge financial support through senior research fellowships from UGC-BSR, UGC, and CSIR respectively. SP thanks G Baskaran and B Kumar for discussions on the experimental data.

\section{References}

[1] Hor Y S, Williams A J, Checkelsky J G, Roushan P, Seo J, $\mathrm{Xu}$ Q, Zandbergen $\mathrm{H} \mathrm{W}$, Yazdani A, Ong N P and Cava R J 2010 Phys. Rev. Lett. 104057001

[2] Kriener M, Segawa K, Ren Z, Sasaki S, Wada S, Kuwabata S and Ando Y 2011 Phys. Rev. B 84054513

[3] Balakrishnan G, Bawden L, Cavendish S and Lees M R 2013 Phys. Rev. B 87140507
[4] Wilczek F 2009 Nat. Phys. 5614

[5] Fu L and Kane C L 2008 Phys. Rev. Lett. 100096407

[6] Zhong R D, Schneeloch J A, Shi X Y, Xu Z J, Zhang C, Tranquada J M, Li Q and Gu G D 2013 Phys. Rev. B 88020505

[7] Maurya V K, Shruti, Shrivastava P and Patnaik S 2014 Europhys. Lett. 10837010

[8] Tanaka Y, Ren Z, Sato T, Nakayama K, Souma S, Takahashi T, Segawa K and Ando Y 2012 Nat. Phys. 8800

[9] Hsieh H, Lin H, Liu J, Duan W, Bansil A and Fu L 2012 Nat. Commun. 3982

[10] Kirshenbaum K, Syers P S, Hope A P, Butch N P, Jeffries J R, Weir S T, Hamlin J J, Maple M B, Vohra Y K and Paglione J 2013 Phys. Rev. Lett. 111087001

[11] Kong P P et al 2013 J. Phys.: Condens. Matter 25362204

[12] Zhang S J, Zhang J L, Yu X H, Zhu J and Kong P P 2012 J. Appl. Phys. 111112630

[13] Sachdev S and Keimer B 2011 Phys. Today 6429

[14] Hamlin J J, Jeffries J R, Butch N P, Syers P, Zocco D A, Weir S T, Vohra Y K, Paglione J and Maple M B 2012 J. Phys.: Condens. Matter 24035602

[15] Hor Y S et al 2010 Phys. Rev. Lett. 104057001

[16] Sasaki S, Kriener M, Segawa K, Yada K, Tanaka Y, Sato M and Ando Y 2011 Phys. Rev. Lett. 107217001

[17] Bay T V, Naka T, Huang Y K, Luigjes H, Golden M S and de Visser A 2012 Phys. Rev. Lett. 108057001

[18] Zhou D, Li Q, Ma Y, Cui Q and Chen C 2013 J. Phys. Chem. C 11712266

[19] Wang H, Wang H, Liu H, Lu H, Yang W, Jia S, Liu X-J, Xie X C, Wei J and Wang J arXiv:1501.00418

[20] Eiling A and Schilling J S 1981 J. Phys. F: Metal Phys. 11623

[21] Takahashi H and Mori N 1995 Studies of High Temperature Superconductors vol 16, ed A V Narlikar (New York: Nova Science) 